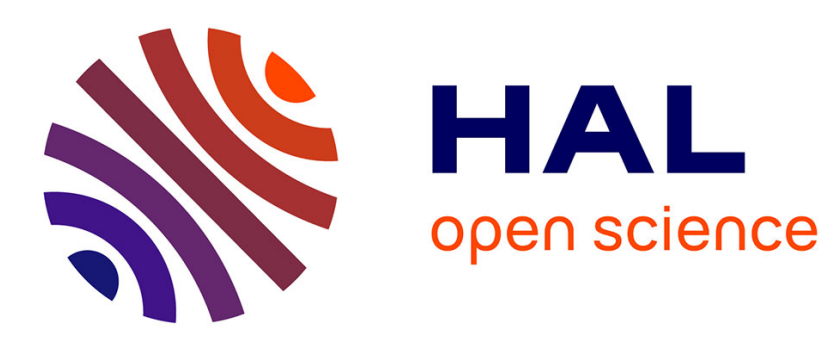

\title{
A stochastic force model for the ballistic-diffusive transition of heat conduction
}

Pier Luca Palla, Giuseppe Patera, Fabrizio Cleri, Stefano Giordano

\section{To cite this version:}

Pier Luca Palla, Giuseppe Patera, Fabrizio Cleri, Stefano Giordano. A stochastic force model for the ballistic-diffusive transition of heat conduction. Physica Scripta, 2020, 95 (7), pp.075703. 10.1088/1402-4896/ab8d56 . hal-02947988

\section{HAL Id: hal-02947988 \\ https://hal.science/hal-02947988}

Submitted on 24 Sep 2020

HAL is a multi-disciplinary open access archive for the deposit and dissemination of scientific research documents, whether they are published or not. The documents may come from teaching and research institutions in France or abroad, or from public or private research centers.
L'archive ouverte pluridisciplinaire HAL, est destinée au dépôt et à la diffusion de documents scientifiques de niveau recherche, publiés ou non, émanant des établissements d'enseignement et de recherche français ou étrangers, des laboratoires publics ou privés. 


\title{
A stochastic force model for the ballistic-diffusive transition of heat conduction
}

\author{
Pier Luca Palla ${ }^{1}$, Giuseppe Patera ${ }^{2}$, Fabrizio Cleri ${ }^{1}$, \\ Stefano Giordano ${ }^{1}$ \\ ${ }^{1}$ Univ. Lille, CNRS, ISEN, Univ. Valenciennes, UMR 8520 - IEMN - Institute \\ of Electronics, Microelectronics and Nanotechnology, F-59000 Lille, France, \\ 2 Univ. Lille, CNRS, UMR 8523 - PhLAM - Laboratory of Physics of Lasers, \\ Atoms and Molecules, F-59000 Lille, France \\ E-mail: stefano.giordano@univ-lille.fr
}

\begin{abstract}
We theoretically study the heat conduction in a harmonic chain with a stochastic force field, in contact with two Langevin thermal baths at different temperatures. In particular, we investigate the interplay between the thermal baths properties and the size of the system. To this aim, we introduce a stochastic force field, which is simple enough to be energy-conserving for each particle of the chain, but sufficiently aleatory to induce the ballistic to diffusive transition in the conductive behavior of the chain. When this stochastic force field is absent, we observe a ballistic behavior strongly dependent on the characteristic collision frequency of the thermal baths for any size of the system. On the other hand, when the stochastic force field is activated, the diffusive behavior is established and the effect of the thermal baths is removed in the thermodynamic limit.
\end{abstract}

Keywords: heat conduction; stochastic methods; transport processes Submitted to: Phys. Scr. 


\section{Introduction}

While the thermal conduction in macroscopic objects is well described by the Fourier diffusive law, the thermal transport in low dimensional and nanostructured materials can show a ballistic behavior, which have potential applications in nanodevices thermal management [1], thermoelectric efficiency [2], and quantum informatics [3]. The transition between diffusive and ballistic regimes of heat transport [4-6] has been experimentally observed in several systems including silicon nanowires [7-10], doped silicon nanowires [11], carbon or silicon nanotubes [12, 13], silicon membranes [14-17], rarefied air chambers [18], liquids [19], and graphene ribbons [20,21]. In spite of these recent refined measurements, since the time of the Fourier's early work [22], the microscopic foundations of heat conduction still remain partially unexplained. In particular, the theoretical interpretation of the ballistic to diffusive transition represents a crucial problem of the out-of-equilibrium statistical mechanics.

From the historical point of view, since the 19th century, the advancement of the out-of-equilibrium statistical mechanics received a great impulse by the observation of the Brownian motion [23, 24] and by its thoroughgoing interpretations of Einstein $[25,26]$ and Smoluchowski [27]. These investigations led Perrin to accurately measure the Avogadro number [28], definitively confirming the atomistic hypothesis. In this context, the Langevin stochastic differential equation was successufully stated [29], and analysed through the Fokker-Planck methodology [30-32]. More recently, Sekimoto defined the concept of heat for a given fluctuating trajectory $[33,34]$, leading to the origin of the stochastic thermodynamics. At the same time, the concepts of entropy and entropy production have been generalized to deal with the Brownian motion $[35,36]$, allowing for the demonstration of the laws of thermodynamics [37-40], and other fluctuation theorems [41-44], on the base of the Langevin postulates.

The development of these concepts is at the base of the understanding of the thermal transfer in solids and nanomaterials. Indeed, the simplest introduced idealization of a heat conducting system is a chain of $N$ masses in contact at its extremities with two thermal baths at different temperatures. On the one hand, if the chain is characterized by purely harmonic interactions, heat diffusion (Fourier) is not established, and a ballistic regime with thermal conductivity $\kappa \propto N$ is theoretically observed [45]. In this case, the system has been analyzed in a stationary non equilibrium state, by obtaining, through the Langevin-FokkerPlanck formalism, the closed form expression for the covariance matrix of the positions of particles in the chain [45]. On the other hand, in the ballistic regime, the chain elements transfer energy in each mode without interactions all along the length, and the only exchange of energy between modes takes place at the boundaries through the interaction with the thermal baths. To obtain a diffusive heat transfer, characterized by a finite value of $\kappa$ for $N \rightarrow \infty$, some form of anharmonicity, disorder and/or randomness must be included in the system [46], in order to foster the energy exchange among the modes. Since this is a scale-dependent process (see the finite mean free path of phonons in a solid), deviations from the ballistic regime would grow with the system size, eventually yielding the expected diffusive behavior in the thermodynamic limit.

However, the minimal conditions for observing Fourier conduction in an anharmonic chain are not well defined $[46,47]$. The related problem of ergodicity in a chain of anharmonic oscillators was first studied by Fermi, Pasta and Ulam (FPU) in a seminal numerical experiment [48], with the well-known result that equipartition was not observed (see Ref. [49] for the long time scale of the FPU problem). More recently, it has been proved that the strong nonlinearity regime entails the so-called anomalous diffusion (with $\kappa \propto N^{\xi}$, $0<\xi<1$ ) [50-54]. From then on, several ad hoc models have been proposed in the attempt to reproduce the diffusive conduction in simple systems. Without any pretension to being exhaustive, one could cite: the ding-a-ling model [55]; the ding-dong model $[56,57]$; the Lorentz gas model [58]; the FrenkelKontorova model $[59,60]$; the anharmonic chain with weak interparticle potential [61]; the 2D harmonic lattice model with missing bond defects [62]; the chain of coupled rotators [63]; the pinned anharmonic chain [64]; the harmonic chain with inner stochastic reservoirs obeying the self-consistent condition [65-67]; the harmonic chain with graded mass distribution [68]; the lattice billiard [69]; the chain with conservative stochastic dynamics [70-77]. In all these cases, more or less complex perturbations to the initial harmonic chain have been introduced to force the energy exchange between the mode and to eventually generate a diffusive heat transport. However, depending on the working conditions, the thermal bath features may influence the value of $\kappa$, thus weakening the concept of thermal conductivity as an intrinsic property of the system $[45,46,78]$. For instance, $\kappa$ depends on the collision frequency when Langevin thermal baths are used $[45,46]$. Also, it has been proved that the thermal bath spectral properties can change the anomalous diffusive response of a system [78].

In this paper, we attempt to clarify this issue by considering a quite simple stochastic perturbation of the harmonic force field. We show that switching 


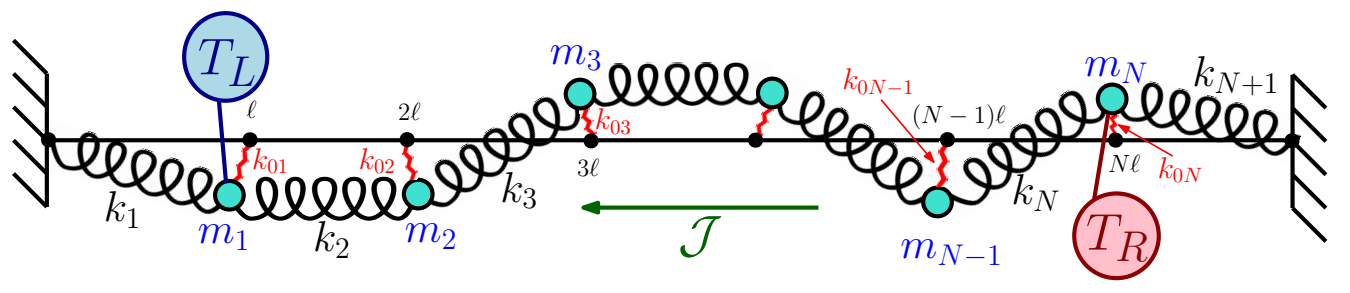

Figure 1. (color online) Structure of the chain conducting the heat flow $\mathcal{J}$ between the reservoirs at temperatures $T_{L}$ and $T_{R}>T_{L}$.

on the perturbation describes the transition from the ballistic to the diffusive regime for increasing values of $N$, thus capturing the scale effects of the heat conduction in terms of the interplay between: (i) the collision frequency $\beta$ of the Langevin thermal baths, and (ii) the intensity $\mu$ of the stochastic perturbation. The final result is a scale-dependent thermal conductivity $\kappa=a N /(b+\mu N)$, with $a$ and $b$ parameters independent of $N$ and $\mu$, and only $b$ depending on the collision frequency $\beta$. The two limiting cases are: $\mu=0$ (zero stochastic perturbation), the conductivity is purely ballistic and linear with $N$, and a function of the thermal bath properties (through $b$ ); and the thermodynamic limit $(N \rightarrow \infty)$ with $\mu \neq 0$, which leads to diffusive heat transfer with a finite conductivity $\kappa^{\text {diff }}=a / \mu$, thereby assigning a well-defined intrinsic meaning to the concept of thermal conductivity of a macroscopic system. We also study the relation between the elastic constants of the harmonic interactions of the chain and its final heat conductivity. The origin of the ballistic-diffusive transition is finally discussed with reference to possible applications to nanoscience and nanotechnology.

\section{Stochastic force field}

In the following we will consider a system of $N$ particles of given mass linked by harmonic springs, in contact with two "thermostats", and subject to a perturbation inducing non-linearity of the response (see Fig.1). Since a nonlinear model cannot be studied analytically, we introduce an effective stochastic perturbation to mimic the chaotic effects arising in a real nonlinear system. A minimal example of such perturbation can be constructed as the cross product of the instantaneous velocity $\vec{v}(t)$ and a random noise $\vec{n}(t)$. By construction, such a "vector-velocity" perturbation randomly modifies the direction of the velocity of a particle while conserving its modulus. While the twodimensional version of this stochastic process has been introduced in the literature $[77,79]$, here we analyze and discuss its three-dimensional implementation. The (Langevin) stochastic dynamics of a single particle would be written:

$\frac{\mathrm{d} \vec{v}}{\mathrm{~d} t}=-\gamma \vec{v}+\sqrt{\mu} \vec{v} \wedge \vec{n}$,

with $\mu$ the amplitude of the perturbation and $\gamma$ a friction coefficient. The noise can be taken as Gaussian, with the standard properties of expectation value equal to zero for the first moment $\mathbb{E}\left\{n_{j}(t)\right\}=0$, and $\mathbb{E}\left\{n_{i}\left(t_{1}\right) n_{j}\left(t_{2}\right)\right\}=2 \delta_{i j} \delta\left(t_{1}-t_{2}\right)$ for the second moment (it is the so-called white or uncorrelated Gaussian noise) [80-82].

This stochastic differential equation with multiplicative noise for the variable $\vec{v}(t)$ is not completely defined mathematically. It gets a precise meaning only after choosing a convention for the stochastic integrals, by specifying a parameter $0 \leq \alpha \leq 1$ that defines the point at which an integrated function is evaluated in each interval of the adopted Riemann sum [80-82]. More specifically, if we consider an arbitrary system of differential equations $\frac{d \vec{x}}{d t}=f(\vec{x}, t)$, we can write on a time interval $\Delta t$ the evolution $\Delta \vec{x}=\int_{t}^{t+\Delta t} f(\vec{x}, t) d t$, where $\Delta \vec{x}=\vec{x}(t+\Delta t)-\vec{x}(t)$. And the integral can be approximated through the expression $\Delta \vec{x}=f(\vec{x}+\alpha \Delta \vec{x}, t) \Delta t$, where $\alpha$ determines the position at which we calculate the function $f$ in the interval $(t, t+\Delta t)$. Now, the important point is that if $f$ is a deterministic function, $\alpha$ does not influence the result for small values of $\Delta t$; on the other hand, if $f$ has a stochastic character, $\alpha$ may strongly affect the result, in particular when multiplicative white noises are present as in our model [80-82]. It is important to remember that the uncorrelated white noise is an idealization of real physical processes, which we interpret as fluctuations. From a physical point of view, the FiskStratonovich approach, with $\alpha=1 / 2$ [83, 84], should be adopted since it can be obtained as the limiting process of a coloured noise (with finite energy) towards an uncorrelated white one (with diverging energy) [80-82]. However, for the sake of generality, in the following development we maintain an arbitrary value of $\alpha$ (including the Itô $(\alpha=0)$ [85] and the Hänggi-Klimontovich $(\alpha=1)[86,87]$ particular cases), and will later show that $\alpha$ does not influence the system response (for further details on the stochastic interpretation see also Ref. [88]). In particular, $\alpha$ does not influence the thermal conductivity of the system under investigation. 
In our model, the precise value of this parameter is linked to the amplitude of the perturbation, $\mu$, and to the friction coefficient, $\gamma$. By writing down the FokkerPlanck equation [80-82] for the probability density $W(\vec{v}, t)$ of the velocity vector $\vec{v}$,

$$
\begin{aligned}
\frac{\partial W}{\partial t}= & \gamma \frac{\partial}{\partial v_{i}}\left(v_{i} W\right)+4 \alpha \mu \frac{\partial}{\partial v_{i}}\left(v_{i} W\right) \\
& +\frac{\partial^{2}}{\partial v_{i} \partial v_{j}}\left[\mu\left(\delta_{i j} v_{p} v_{p}-v_{i} v_{j}\right) W\right],
\end{aligned}
$$

associated to Eq.(1), we determine the time evolution of $\mathbb{E}\{\vec{v} \cdot \vec{v}\}$ and $\mathbb{E}\{\sqrt{\vec{v} \cdot \vec{v}}\}$ as:

$$
\begin{gathered}
\frac{\mathrm{d}}{\mathrm{d} t} \mathbb{E}\{\vec{v} \cdot \vec{v}\}=2[-\gamma+2(1-2 \alpha) \mu] \mathbb{E}\{\vec{v} \cdot \vec{v}\}, \\
\frac{\mathrm{d}}{\mathrm{d} t} \mathbb{E}\{\sqrt{\vec{v} \cdot \vec{v}}\}=[-\gamma+2(1-2 \alpha) \mu] \mathbb{E}\{\sqrt{\vec{v} \cdot \vec{v}}\},
\end{gathered}
$$

and we conclude that the dynamics can simultaneously conserve $\mathbb{E}\{\vec{v} \cdot \vec{v}\}$ and $\mathbb{E}\{\sqrt{\vec{v} \cdot \vec{v}}\}$ with the choice $\gamma=$ $2(1-2 \alpha) \mu$. It is interesting to observe that under the Stratonovich interpretation $(\alpha=1 / 2)$ [84], the viscous dissipative term in Eq.(1) vanishes $(\gamma=$ $0)$. This can be understood by observing that the usual rules of calculus remain unchanged within the Stratonovich interpretation of the stochastic calculus. Therefore, through the scalar product of Eq.(1) and $\vec{v}$, we directly obtain the time-invariance of $\mathbb{E}\{\vec{v} \cdot \vec{v}\}$ with $\gamma=0$. However, the other stochastic interpretations require the dissipative term with the pertinent collision frequency $\gamma=2(1-2 \alpha) \mu$.

This choice has, moreover, much wider implications. In fact, by adopting a stochastic calculus based on the as-defined $\alpha$, the complete, generalized Langevin equation for a particle embedded in a potential energy field $V(\vec{r})$, in contact with a Langevin thermal bath, and subjected to the force in Eq.(1), can be written as:

$\frac{\mathrm{d} \vec{r}}{\mathrm{~d} t}=\frac{\vec{p}}{m}$,

$\frac{\mathrm{d} \vec{p}}{\mathrm{~d} t}=-\frac{\partial V}{\partial \vec{r}}-\gamma \vec{p}+\sqrt{\mu} \vec{p} \wedge \vec{n}-\beta \vec{p}+\sqrt{\beta K_{b} T m} \vec{n}_{a}$.

The corresponding Fokker-Planck equation [80-82], governing the probability density $W(\vec{r}, \vec{p}, t)$, can be eventually obtained as:

$$
\begin{aligned}
\frac{\partial W}{\partial t}= & -\frac{p_{i}}{m} \frac{\partial W}{\partial x_{i}}+\beta \frac{\partial}{\partial p_{i}}\left(p_{i} W\right)+\frac{\partial V}{\partial x_{i}} \frac{\partial W}{\partial p_{i}} \\
& +\beta K_{b} T m \frac{\partial^{2} W}{\partial p_{i} \partial p_{i}}+(\gamma+4 \alpha \mu) \frac{\partial}{\partial p_{i}}\left(p_{i} W\right) \\
& -6 \mu W-4 \mu p_{i} \frac{\partial W}{\partial p_{i}}+\mu\left(\delta_{i j} p_{k} p_{k}-p_{i} p_{j}\right) \frac{\partial^{2} W}{\partial p_{i} \partial p_{j}}
\end{aligned}
$$

where the first four terms correspond to the classical Klein-Kramers equation [89-91] and the others to the stochastic perturbation. For $\gamma=0$ and $\mu=$ 0 , the system obviously attains the canonical Gibbs distribution at large times $[82,91,92]$. However, we proved through Eq.(7) that this is true also for $\gamma \neq 0$ and $\mu \neq 0$, provided that $\gamma=2(1-2 \alpha) \mu$. This means that the stochastic perturbation here introduced does not modify the thermodynamic equilibrium, if it exists. Equivalently, it implies that the effect of the stochastic perturbation could only be observed in the non-equilibrium regime. This is a central point for our application to the heat conduction problem.

Interestingly enough, to better understand the effect of the introduced stochastic perturbation in the out-of-equilibrium evolution, we can investigate the Brownian diffusion of a free particle (we let $V=0$ and $\gamma=2(1-2 \alpha) \mu$ in Eqs. (5), (6) and (7)). Through the Fokker-Planck formalism, we obtain the evolution of the average values of coordinates and momenta, namely $(\mathrm{d} / \mathrm{d} t) \mathbb{E}\{\vec{r}\}=\frac{1}{m} \mathbb{E}\{\vec{p}\}$ and $(\mathrm{d} / \mathrm{d} t) \mathbb{E}\{\vec{p}\}=$ $-(\beta+2 \mu) \mathbb{E}\{\vec{p}\}$, and of the covariances, as follows

$$
\begin{aligned}
\frac{\mathrm{d}}{\mathrm{d} t} \mathbb{E}\left\{x_{n} x_{m}\right\}= & \frac{1}{m} \mathbb{E}\left\{x_{m} p_{n}\right\}+\frac{1}{m} \mathbb{E}\left\{x_{n} p_{m}\right\}, \\
\frac{\mathrm{d}}{\mathrm{d} t} \mathbb{E}\left\{x_{n} p_{m}\right\}= & \frac{1}{m} \mathbb{E}\left\{p_{n} p_{m}\right\}-(\beta+2 \mu) \mathbb{E}\left\{x_{n} p_{m}\right\}, \\
\frac{\mathrm{d}}{\mathrm{d} t} \mathbb{E}\left\{p_{n} p_{m}\right\}= & -2(\beta+3 \mu) \mathbb{E}\left\{p_{n} p_{m}\right\}+2 \beta K_{b} T \delta_{n m} \\
& +2 \mu \delta_{n m} \mathbb{E}\left\{p_{k} p_{k}\right\} .
\end{aligned}
$$

By solving these differential equations (starting from the initial deterministic condition $\vec{r}(0)=0$ and $\vec{p}(0)=$ 0 ), we easily obtain that $\mathbb{E}\left\{x_{n} x_{m}\right\}=\mathbb{E}\left\{x_{n} p_{m}\right\}=$ $\mathbb{E}\left\{p_{n} p_{m}\right\}=0$ when $n \neq m$, and

$$
\begin{aligned}
\mathbb{E}\left\{x_{n}^{2}\right\}= & \frac{2 K_{b} T t}{m(\beta+2 \mu)}-\frac{K_{b} T}{m \beta(\beta-2 \mu)} \mathrm{e}^{-2 \beta t} \\
& +\frac{4 \beta K_{b} T}{m(\beta-2 \mu)(\beta+2 \mu)^{2}} \mathrm{e}^{-(\beta+2 \mu) t} \\
& +\frac{(3 \beta+2 \mu) K_{b} T}{m \beta\left(\beta^{2}+4 \beta \mu+4 \mu^{2}\right)} \\
\mathbb{E}\left\{x_{n} p_{n}\right\}= & \frac{K_{b} T}{\beta+2 \mu}+\frac{K_{b} T}{\beta-2 \mu} \mathrm{e}^{-2 \beta t} \\
& -\frac{2 \beta K_{b} T}{(\beta-2 \mu)(\beta+2 \mu)} \mathrm{e}^{-(\beta+2 \mu) t} \\
\mathbb{E}\left\{p_{n}^{2}\right\}= & m K_{b} T\left(1-\mathrm{e}^{-2 \beta t}\right)
\end{aligned}
$$

It means that the diffusion coefficient $D=\frac{2 K_{b} T}{m(\beta+2 \mu)}$, appearing in the relation $\mathbb{E}\left\{x_{n}^{2}\right\}=D t$ (see Eq.(11) for large $t$ ), is simply modified with respect to the case with $\mu=0$ by substituting $\beta$ with $\beta+2 \mu$. Hence, the Stokes law assumes the form $m(\beta+2 \mu)=6 \pi \eta R$, where $\eta$ is the fluid viscosity and $R$ the radius of the diffusing particle [82]. From Eq.(13), we also deduce that the out-of-equilibrium evolution of the kinetic energy is not influenced by the elastic collisions.

\section{Heat conduction in the mass-spring chain}

After discussing these key premises and their implications, we can now move to the study of the complete 
system represented in Fig.1. We consider a chain of particles, with positions $\vec{r}_{i}$ and momenta $\vec{p}_{i}$, described by the generalized Langevin equations $(i=1, \ldots, N)$ :

$$
\begin{aligned}
\frac{\mathrm{d} \vec{r}_{i}}{\mathrm{~d} t}= & \frac{\vec{p}_{i}}{m_{i}}, \\
\frac{\mathrm{d} \vec{p}_{i}}{\mathrm{~d} t}= & -k_{i}\left(\vec{r}_{i}-\vec{r}_{i-1}\right)-k_{i+1}\left(\vec{r}_{i}-\vec{r}_{i+1}\right) \\
& -k_{0 i}\left(\vec{r}_{i}-\vec{r}_{0 i}\right)-\gamma_{i} \vec{p}_{i}+\sqrt{\mu_{i}} \vec{p}_{i} \wedge \vec{n}_{i} \\
& +\delta_{i 1}\left(-\beta \vec{p}_{1}+\sqrt{\beta K_{b} T_{L} m_{1}} \vec{n}_{L}\right) \\
& +\delta_{i N}\left(-\beta \vec{p}_{N}+\sqrt{\beta K_{b} T_{R} m_{N}} \vec{n}_{R}\right),
\end{aligned}
$$

where $\vec{r}_{0 i}=(0,0, i \ell)$ are the unperturbed positions $(i=1, \ldots, N), \vec{r}_{0}=(0,0,0), \vec{r}_{N+1}=(0,0,(N+$ 1) $\ell$ ) define the two extremes of the chain, $\beta$ is the Langevin collision frequency, $\vec{n}_{i}(i=1, \ldots, N)$ are the multiplicative noises, $\vec{n}_{L}$ and $\vec{n}_{R}$ are the additive noises of the left and right Langevin thermal baths with temperatures $T_{L}$ and $T_{R}$, respectively, applied only to the particles at extremities. The choice $\gamma_{i}=$ $2(1-2 \alpha) \mu_{i}$ ensures that all the properties described above for the single particle case are also retained for the elements of the chain. Furthermore, the masses $m_{i}$ may be subjected to an external linear pinning $\left(k_{0 i}\right)$, in addition to nearest neighbor linear interactions $\left(k_{i}\right)$. In this model, the particles move in the whole 3D space and this is useful for further generalizations to arbitrary (possibly heterogeneous) 2D or 3D lattices.

To define the heat flow $\mathcal{J}$ between the two ends of the system ("left" and "right"), we perform the scalar product between $\vec{p}_{1} / m_{1}$ (or $\left.\vec{p}_{N} / m_{N}\right)$ and the Newton equation of the first (or last) particle of the chain, and identify the heat rates through the relations:

$$
\frac{\mathrm{d} Q_{D}}{\mathrm{~d} t}=\mathbb{E}\left\{-\beta \frac{\vec{p}_{d} \cdot \vec{p}_{d}}{m_{d}}+\sqrt{\frac{\beta K_{b} T_{D}}{m_{d}}} \vec{n}_{D} \cdot \vec{p}_{d}\right\}
$$

(with $D=L, d=1$ on the left; or $D=R$, $d=N$ on the right), consistently with the Sekimoto interpretation of the heat transfer along the trajectory of a stochastic system $[33,34,93]$. By averaging the result under nonequilibrium stationary state (NESS), and taking into account that the power of the stochastic perturbation is zero, we get:

$\frac{\mathrm{d} Q_{L}}{\mathrm{~d} t}=\frac{k_{01}+k_{1}+k_{2}}{m_{1}} \mathbb{E}\left\{\vec{r}_{1} \cdot \vec{p}_{1}\right\}-\frac{k_{2}}{m_{1}} \mathbb{E}\left\{\vec{r}_{2} \cdot \vec{p}_{1}\right\}$,

and

$$
\begin{aligned}
\frac{\mathrm{d} Q_{R}}{\mathrm{~d} t}= & \frac{k_{0 N}+k_{N}+k_{N+1}}{m_{N}} \mathbb{E}\left\{\vec{r}_{N} \cdot \vec{p}_{N}\right\} \\
& -\frac{k_{N}}{m_{N}} \mathbb{E}\left\{\vec{r}_{N-1} \cdot \vec{p}_{N}\right\} .
\end{aligned}
$$

Of course, at NESS, $\mathcal{J}=\frac{\mathrm{d} Q_{L}}{\mathrm{~d} t}=-\frac{\mathrm{d} Q_{R}}{\mathrm{~d} t}$. Therefore, the thermal characterization of the chain can be carried out from Eqs.(17) and (18) if we are able to determine the covariances $\mathbb{E}\left\{\vec{r}_{i} \cdot \vec{p}_{j}\right\}$ between coordinates and momenta at stationary state.

To simplify the treatment, we define a state vector $\mathcal{X}=\left(\vec{r}_{1}, \ldots, \vec{r}_{N}, \vec{p}_{1}, \ldots, \vec{p}_{N}\right)^{T} \in \mathbb{R}^{6 N}$, by which Eqs.(14) and (15) can be rewritten as:

$\frac{\mathrm{d}}{\mathrm{d} t} \mathcal{X}=\left(\mathcal{A}+\sum_{\xi=1}^{3 N} n_{\xi} \mathcal{B}_{\xi}\right) \mathcal{X}+\mathcal{C N}_{a}+\mathcal{V}$,

where $\mathcal{A}$ and $\mathcal{B}_{\xi}$ are $6 N \times 6 N$ matrices; $\mathcal{C}$ is a $6 N \times 6$ matrix and $\mathcal{V} \in \mathbb{R}^{6 N} ; \mathcal{N}_{a}=\left(\vec{n}_{L}, \vec{n}_{R}\right)^{T} \in \mathbb{R}^{6}$ describes the additive noises; moreover, $n_{\xi}$ are the components of the multiplicative noise vector $\left(\vec{n}_{1}, \ldots, \vec{n}_{N}\right)^{T}$. The entries of these matrices and vectors can be directly obtained by comparison with the structure of Eqs.(14) and (15).

The final stochastic differential equation describes a generalization of the Ornstein-Uhlenbeck process with multiplicative noises. Although the multiplicative noises generally prevent the solutions to be a Gaussian process, we can obtain a closed set of linear equations for the average state $\mathbb{E}\{\mathcal{X}\}$ and for the covariance matrix $\Lambda=\mathbb{E}\left\{\mathcal{X} \mathcal{X}^{T}\right\}$. Indeed, the Fokker-Planck equation [80-82] for $W(\mathcal{X}, t)$, associated with Eq.(19), can be written as

$$
\begin{aligned}
\frac{\partial W}{\partial t}= & -\vec{\nabla} \cdot[(\mathcal{S} \mathcal{X}+\mathcal{V}) W]+\vec{\nabla} \cdot\left(\mathcal{C C}^{T} \vec{\nabla} W\right) \\
& +\vec{\nabla} \cdot\left[\sum_{\xi} \mathcal{B}_{\xi}^{2} \mathcal{X} W\right]+\vec{\nabla} \cdot\left[\sum_{\xi} \mathcal{B}_{\xi} \mathcal{X} \operatorname{Tr}\left(\mathcal{B}_{\xi}\right) W\right] \\
& +\vec{\nabla} \cdot\left[\sum_{\xi} \mathcal{B}_{\xi} \mathcal{X}\left(\vec{\nabla} W \cdot \mathcal{B}_{\xi} \mathcal{X}\right)\right],
\end{aligned}
$$

and allows to obtain

$\frac{\mathrm{d}}{\mathrm{d} t} \mathbb{E}\{\mathcal{X}\}=\mathcal{S} \mathbb{E}\{\mathcal{X}\}+\mathcal{V}$,

$\frac{\mathrm{d}}{\mathrm{d} t} \Lambda=\mathcal{S} \Lambda+\Lambda \mathcal{S}^{T}+2 \sum_{\xi} \mathcal{B}_{\xi} \Lambda \mathcal{B}_{\xi}^{T}+2 \mathcal{C C} \mathcal{C}^{T}+\mathcal{T}$,

where $\mathcal{S}=\mathcal{A}+2 \alpha \sum_{\xi} \mathcal{B}_{\xi}^{2}$, and $\mathcal{T}=\mathcal{V} \mathbb{E}\{\mathcal{X}\}^{T}+$ $\mathbb{E}\{\mathcal{X}\} \mathcal{V}^{T}$. We remark that if $\mathcal{B}_{\xi}=0 \forall \xi$, the classical results concerning the Ornstein-Uhlenbeck process [94, 95] are perfectly retrieved. Anyway, if one is interested in the NESS, characterized by $(\mathrm{d} / \mathrm{d} t) \mathbb{E}\{\mathcal{X}\}=0$ and $(\mathrm{d} / \mathrm{d} t) \Lambda=0$, the covariances can be directly calculated as the solution of a sparse linear system with $36 N^{2}$ unknowns. It assumes the final form

$$
\begin{aligned}
0= & \mathcal{S} \Lambda+\Lambda \mathcal{S}^{T}+2 \sum_{\xi} \mathcal{B}_{\xi} \Lambda \mathcal{B}_{\xi}^{T} \\
& +2 \mathcal{C} \mathcal{C}^{T}-\mathcal{V} \mathcal{V}^{T} \mathcal{S}^{-T}-\mathcal{S}^{-1} \mathcal{V} \mathcal{V}^{T}
\end{aligned}
$$

whose solution for $\Lambda$ can be found through the numerical method discussed in the Appendix.

For the purpose of comparing our result for $\mathcal{J}$ with the classical diffusive constitutive equation $\mathcal{J}=$ 


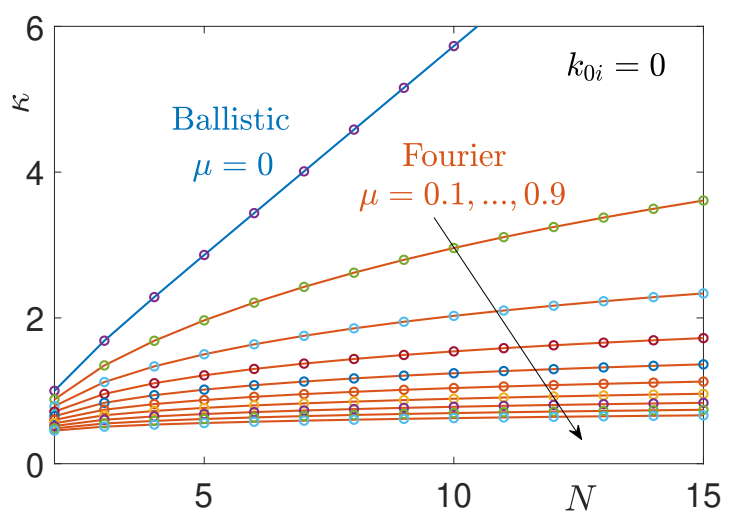

Figure 2. (color online) Plot of $\kappa$ versus $N$ showing the transition between ballistic and diffusive (Fourier) conduction. We adopted the parameters: $k_{i}=1 \forall i, m_{i}=1 \forall i, \beta=1$, $K_{b} T_{L}=1, K_{b} T_{R}=2, k_{0 i}=0 \forall i$. All quantities are in arbitrary units.

$-\kappa \frac{\partial T}{\partial x}$, in our chain we define the thermal conductivity $\kappa=\mathcal{J} N \ell /\left(T_{L}-T_{R}\right)$, where $\mathcal{J}$ is calculated through Eqs.(17) and (18). We remark that the calculation of $\mathcal{J}$ and $\kappa$ based on the method of the covariances is exact.

Figure 2 shows the behavior of $\kappa$ vs. $N$. The conductivity is clearly ballistic (linear response of $\kappa$ versus $N$ ) for $\mu=0$, and goes into diffusive for increasing values of $\mu$ ( $\kappa$ becoming independent of $N$ for long chains). While there is no finite critical value of $\mu$ required for this transition, for small values of $\mu$ it is necessary to use longer chains to observe the transition.

In Fig.3, we show the distribution of kinetic energy (i.e., temperature) along the chain for both the ballistic and diffusive behavior. It can be observed that for the ballistic case no temperature gradient is created between the reservoirs and, accordingly, the thermal conductivity is diverging for increasing $N$. On the other hand, at increasing values of $\mu$ the temperature tends to become linear within the chain, thereby implying that the thermal flux must become Fourierlike, $\mathcal{J} \propto N^{-1}$; as noted above, there is no critical value of the perturbation, for large enough $N$. We also note that the kinetic energies of the first and last particles are coherent with the temperatures of the lateral Langevin thermal baths, being $\frac{1}{2 m_{1}} \vec{p}_{1} \cdot \vec{p}_{1}=$ $\frac{3}{2} K_{b} T_{L}$ on the left and $\frac{1}{2 m_{N}} \vec{p}_{N} \cdot \vec{p}_{N}=\frac{3}{2} K_{b} T_{R}$ on the right, for large values of $\mu$.

In the conventional, macroscopic analysis of thermal conductivity, the length dependence is usually advocated by "boundary scattering" terms in the Matthiessen's empirical rule $\kappa=\kappa_{\infty} N /(c+N)$. In Fig. 4 we plot the same data of Fig. 2 as $\mu \kappa$ vs. $\mu N$ for both the pinned $\left(k_{0 i} \neq 0\right)$ and unpinned $\left(k_{0 i}=0\right)$ linear chain, and all the results for any value of $\mu$ and

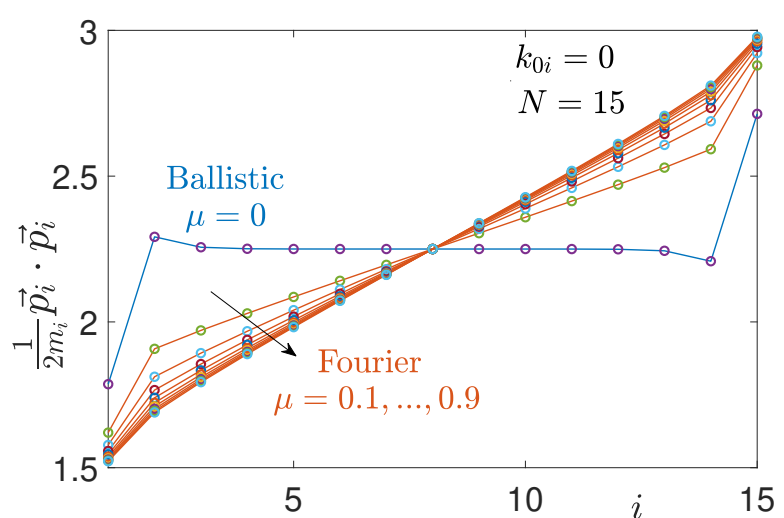

Figure 3. (color online) Plot of the kinetic energy $\frac{1}{2 m_{i}} \vec{p}_{i} \cdot \vec{p}_{i}$ of each particle versus the position $i$ in the chain. We adopted the parameters: $N=15, k_{i}=1 \forall i, m_{i}=1 \forall i, \beta=1, K_{b} T_{L}=1$, $K_{b} T_{R}=2, k_{0 i}=0 \forall i$. All quantities are in arbitrary units.

$N$ collapse on a universal response $\mu \kappa=\phi(\mu N)$ with:

$\phi(z)=\frac{a z}{b+z}$,

that is coherent with the Matthiessen's rule, despite that no empirical assumptions about length-scale effects exist in our model of conductivity. It means that $\kappa=a N /(b+\mu N)$, with $a$ and $b$ parameters independent of $N$ and $\mu$ and depending on the other properties of the system, as discussed below. We underline that this behavior is in good qualitative agreement with most of experimental results concerning the size-dependent behavior of the thermal conductivity in nanosystems [7-21]. It is also important to stress that, as anticipated, the conductivity is independent of the parameter $\alpha$, proving that any particular choice of stochastic calculus is irrelevant to the physics of the system.

To complete this analysis of the thermal conduction we must now examine the role of the thermal baths. In Fig.5, left panel, we plot $\kappa$ for a variable $\beta$, that is the "collision frequency" of the Langevin baths applied at the extremities of the chain. The resulting conductivity strongly depends on $\beta$ showing that, in general, the thermal response depends not only on the properties of the chain, but also on those of the heat baths. This is consistent with earlier investigations $[45,46,78]$, which discussed the role of the spectral properties of the reservoirs on the conduction regimes. The continuous curves in the left panel of Fig.5 represent a fit to the data, through Eq.(24) with a constant value of $a$ (independent of $\beta$ ) and:

$\frac{1}{b}=\frac{1}{b_{0}} \frac{p \beta}{\sqrt{\left(\beta_{0}^{2}-\beta^{2}\right)^{2}+p^{2} \beta^{2}}}$.

This choice of $b$ (a kind of second-order band-pass linear filter, suggested by the bell shape of the data points) is the only one that appears to precisely fit 


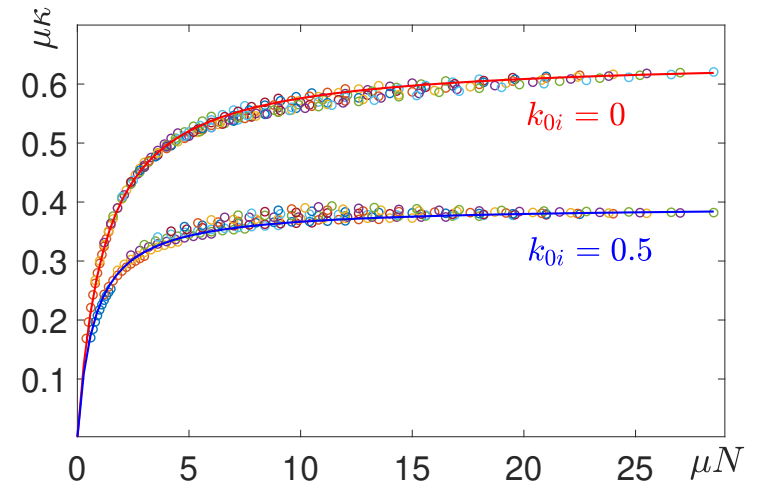

Figure 4. (color online) Plot of $\mu \kappa$ versus $\mu N$ showing the collapse of all data of Fig.2 (circles) to the same response given by $\mu \kappa=\phi(\mu N)$ with $\phi(z)=a z /(b+z)$ (continuous lines). The circles correspond to several results with $5 \leq N \leq 15$ and $0 \leq \mu \leq 2$. We adopted the parameters: $k_{i}=1 \forall i, m_{i}=1 \forall i$, $\beta=1, K_{b} T_{L}=1, K_{b} T_{R}=2, k_{0 i}=0$ (top curve) and $k_{0 i}=0.5$ (bottom curve). All quantities are in arbitrary units.

the analytical results, over more than 4 decades of $\beta$. It appears to suggest that the harmonic force field of the chain can be "tuned" to the frequency of the thermal bath by a sort of damped resonance mechanism, implicit in the second-order character of the equations of motion (Eqs.(14)-(15)).

However, a key feature is that the conductivity becomes independent of $\beta$ in the thermodynamic limit. In the right panel of the same Fig.5, we compare the "reduced conductivity" $\frac{1}{N \mu} \frac{\kappa \mu}{a-\kappa \mu}$ (see Eq.(24)) calculated for various $N$ and $\mu$ values (circles), and the fit with Eq.(25) (continuous line), vs. $\log _{10} \beta$ spanning the very large interval $[-2,2]$; the collapse of all data on a single curve for each harmonic field proves the accuracy of the representation of $\kappa$ by Eqs. (24) and (25). Thus, for $N \rightarrow \infty$ the conductivity takes the value $a / \mu$, independent of $\beta$ and, consequently, independent of the characteristics of the thermal baths. Notably, this holds for both unpinned and pinned systems. The present model is therefore consistent with the general belief that the thermal conductivity is an intrinsic property of any macroscopic system.

We finally investigate the relation between the elastic constant $k_{i}=k \forall i$ of the chain (with $k_{0 i}=$ $0 \forall i$ ) and the resulting heat conduction $\kappa$. To do this, we consider the chain with all the parameters fixed, except for the size $N$ and the elastic constant $k$, which are considered variable over a large range. It is interesting to plot the ratio $k / \kappa$ (elastic constant over heat conductivity) as function of the elastic constant $k$ (see Fig.6). Indeed, we observe a linear behavior of $k / \kappa$ versus $k$ for any value of $N$. It means that $k / \kappa=c k+d$, where $c$ and $d$ are two parameters not depending on the elastic constant $k$. This result allows us to affirm that the dependence of $\kappa$ on $k$ is described
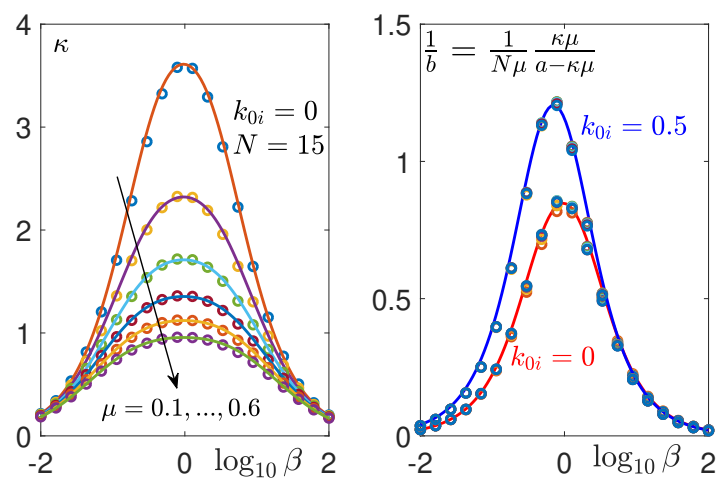

Figure 5. (color online) Effect of the collision frequency $\beta$ on the conduction properties. Left panel: the circles represent $\kappa$ versus $\log _{10} \beta$ for different values of $\mu$. The solid lines are obtained through Eqs.(24) and (25). Right panel: the point represent $\frac{1}{N \mu} \frac{\kappa \mu}{a-\kappa \mu}$ versus $\log _{10} \beta$ showing the collapse of all data (with a constant value of $a,-2 \leq \log _{10} \beta \leq 2,0.1 \leq \mu \leq 0.6$ and $10 \leq N \leq 15)$. The two solid lines are obtained with Eq.(25). We adopted the parameters: $k_{i}=1, m_{i}=1, K_{b} T_{L}=1, K_{b} T_{R}=2$, $k_{0 i}=0$ (unpinned) and $k_{0 i}=0.5$ (pinned). All quantities are in arbitrary units.

by the simple expression $\kappa=\frac{k}{c k+d}$, where $c$ and $d$ are two parameters not depending on the elastic constant $k$. We directly deduce that there is a maximum value of conductivity we can not exceed by simply increasing the elastic constant of the mass-spring chain. In other words $\lim _{k \rightarrow \infty} \kappa=1 / c$, which is a limited quantity, depending on $N$ and on the other parameters of the system. This behavior can be seen in Fig.7, where we plotted the heat conduction versus the elastic constant.

\section{Discussion and conclusions}

In conclusion, the proposed "vector-velocity" stochastic perturbation of the harmonic chain seems to be the simplest choice able to reproduce the ballistic to diffusive transition of thermal conduction, as thoroughly demonstrated by means of an ad hoc generalization of the Ornstein-Ulhenbeck process with multiplicative noises.

The origin of the diffusive behavior of the thermal conduction must be ascribed to the nonlinear character of the interaction forces between the particles (atoms or molecules) of the system under investigation. Indeed, it is well known that a linear (or harmonic) crystal exhibits a ballistic behavior with a thermal conductivity increasing with the size of the system. However, nonlinear systems typically show a complex time evolution that can be only observed through numerical simulations, which prevent a transparent understanding of the underlying physical phenomena. Nevertheless, nonlinear interactions cause a chaotic dynamics of the particles motion. This chaotic regime can be therefore effectively represented by non-thermal 


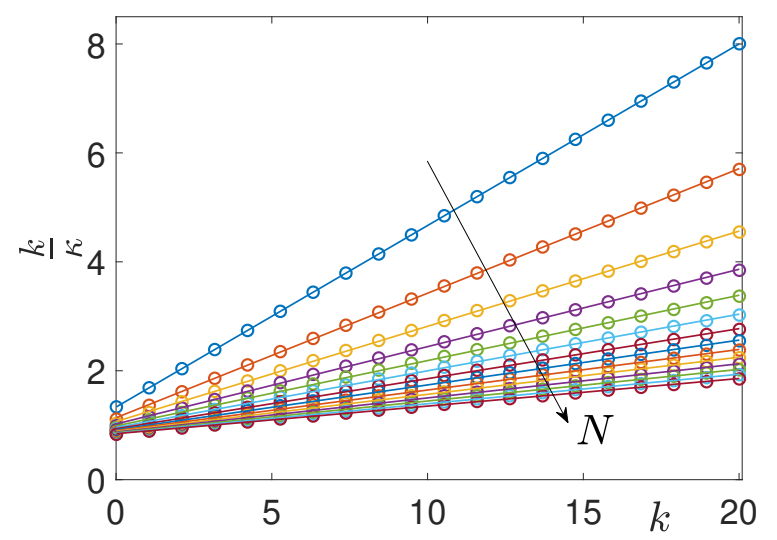

Figure 6. (color online) Plot of the ratio $k / \kappa$ (elastic constant over heat conductivity) as function of the elastic constant $k$ for different values of $N=2, \ldots, 15$ (circles: results obtained through the proposed model; straight lines: least squares approximation of the circles). We adopted the parameters: $k_{i}=k \forall i$ (variable in the range $[0,20]), m_{i}=1 \forall i, \beta=1, K_{b} T_{L}=1, K_{b} T_{R}=2$, $k_{0 i}=0, \mu=0.5$. All quantities are in arbitrary units.

fluctuations mimicking the nonlinear collisions among the system particles (e.g., atoms in a crystalline solids). This is the idea followed in our investigation, where each particle is subjected to the stochastic perturbation defined in Eq.(1). Finally, this random perturbation is the result of the chaotic regime induced by the system nonlinearity. However, in our system, the energy must be exclusively exchanged at the two extremities in contact with the thermal bath. Therefore, the added stochastic perturbation applied to each particle, must be energy-conserving. For this reason, our perturbation is described by a cross product between velocity and noise (similarly to a random magnetic field). It means that the direction of the velocity can fluctuate but not its intensity, and the kinetic energy is conserved. This construction guarantees that no energy enter or leave the system through the inner particles. Hence, we provided evidence that the simplest energy conserving perturbation is able to explain the ballistic-diffusive transition of the heat conduction. Importantly, this result shows that the most relevant property of the perturbation mimicking the particle collisions is its energy-conserving character. Other refined details describing the added perturbation are not relevant (i.e., spectral properties, probabilistic distributions or implementation mechanisms). Indeed, we observed the ballistic-diffusive transition with a classical white Gaussian noise, which stands for the simplest set of assumptions. Moreover, the representation of the nonlinear collisions with a linear stochastic perturbation allows a complete analysis of the problem, which leads to closed form expressions for the behavior of the system. Indeed, the elaboration of the

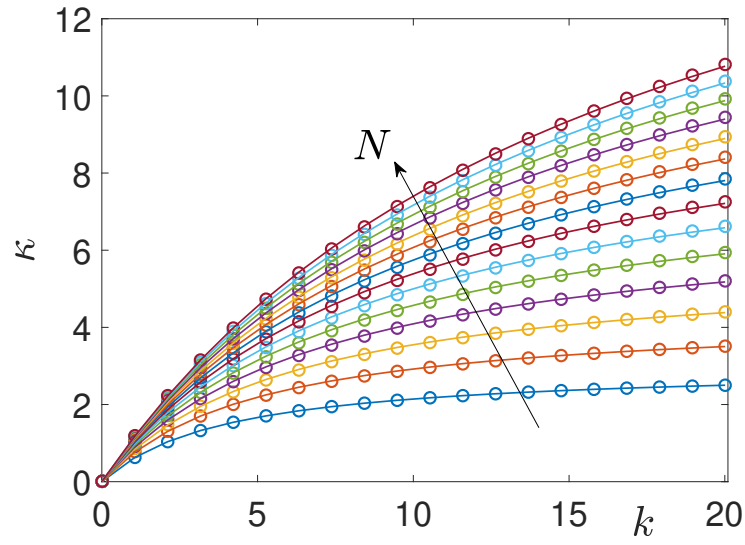

Figure 7. (color online) Heat conductivity $\kappa$ as function of the elastic constant $k$ for different values of $N=2, \ldots, 15$ (circles: results obtained through the proposed model; continuous curves: correspond to the least squares approximations of Fig.6). We adopted the parameters: $k_{i}=k \forall i$ (variable in the range $[0$, $20]), m_{i}=1 \forall i, \beta=1, K_{b} T_{L}=1, K_{b} T_{R}=2, k_{0 i}=0, \mu=0.5$. All quantities are in arbitrary units.

Fokker-Planck formalism for this system leads to a generalization of the Ornstein-Ulhenbeck process with multiplicative noises and to a closed set of equations for the covariances of the particle positions at the stationary state. The knowledge of the covariance matrix can be finally related to the thermal conductivity of the system, which can be therefore easily calculated.

The results show that the ballistic to diffusive transition is observed with increasing size of the system and the process is well described by the socalled Matthiessen's rule. While this scaling law has been previously introduced on the base of empirical arguments, it is here obtained through a rigorous mathematical model. Importantly, the present model sheds light on the role of the thermal baths, proving that the collision frequency influences the transport properties only in the limit of small finite systems, yielding an intrinsic value of the thermal conductivity in the thermodynamic limit. It means that the conductivity is influenced by the thermal reservoirs structure only in small systems and their features can not modify the heat conductivity in very large (ideally infinite) systems. This is reassuring from the point of view of the definition of conductivity, which is generally considered as a material (macroscopic) property, independent of the external conditions. Moreover, we proved that the conductivity is an increasing function of the elastic constant describing the interactions among the system particles. Nevertheless, there is an upper bound of thermal conductivity, which can not be exceeded by simply increasing this elastic constant. Therefore, for an increasing mechanical stiffness of the material, we always observe a consistent saturation of 
the thermal conductivity.

These results may find applications to nanoscale thermal problems, in which one tries to optimize the heat conductivity of a nanosystem between two thermal baths for a finite and small $N$ [96]. Indeed, the present model suggests that it is always possible to tune the thermal baths collision frequency or the harmonic force field of the system to maximize (e.g. for heat dissipation) or minimize (e.g. for thermoelectric optimization) the thermal conductivity.

While being a paradigmatic model for the understanding of the ballistic-diffusive transition, our chain with energy conserving stochastic perturbations should be improved to better represent more realistic situations. One possible analysis should investigate the relationship between the parameters of our system (e.g., $a$ and $b$ of Eq.(24)) and the real physical microscopic features of the material. In our approach, this link is difficult since the stochastic perturbation introduced describes the nonlinear particles collisions, and its intensity $\mu$ is therefore related to the nonlinear part of the interatomic potential energy. Another extension concerns the non-stationary out-of-equilibrium regime of the thermal conduction. Since the Fokker-Planck formalism is not limited to the NESS, our approach can be easily exploited to investigate situations where the reservoirs temperatures, and therefore the heat fluxes, are time varying. It would be also interesting to compare these results with those obtained from a model with true nonlinearities, in order to validate the approach based on the stochastic perturbation and to better understand the relation between our system parameters and the real nonlinear behavior of the particles interactions. To conclude, our model can be applied to the analysis of the thermal conduction of a given system under other externally applied linear effects. For example, it could be generalized to study the effect of a magnetic field on the thermal conductivity.

\section{Appendix: Numerical determination of the covariance matrix}

We discuss here the numerical approach adopted to numerically solve Eq.(23). We define first the Kronecker product of two matrix $\mathcal{A}$ and $\mathcal{B}$ through the block matrix

$\mathcal{A} \otimes \mathcal{B}=\left[\begin{array}{cccc}a_{11} \mathcal{B} & a_{12} \mathcal{B} & a_{13} \mathcal{B} & \ldots \\ a_{21} \mathcal{B} & a_{22} \mathcal{B} & a_{23} \mathcal{B} & \ldots \\ a_{31} \mathcal{B} & a_{32} \mathcal{B} & a_{33} \mathcal{B} & \ldots \\ \vdots & \vdots & \vdots & \ddots\end{array}\right]$

This operation is non-commutative and is useful to convert equations like Eq.(23) to a standard linear system. To do this, we also need to define the vectorization of a matrix. This operation converts a matrix $\mathcal{A}$ into a column vector $\hat{\mathcal{A}}$ by juxtaposing the consecutive rows of the matrix and transposing the result

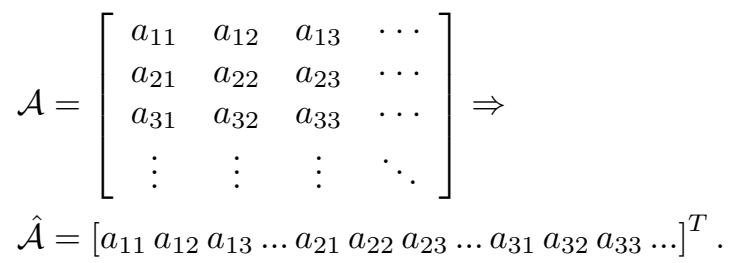

The important relation between Kronecker product and vectorization is given by the following properties

$\mathcal{A}=\mathcal{B C} \Rightarrow \hat{\mathcal{A}}=(\mathcal{B} \otimes \mathcal{I}) \hat{\mathcal{C}}=\left(\mathcal{I} \otimes \mathcal{C}^{T}\right) \hat{\mathcal{B}}$,

$\mathcal{Z}=\mathcal{A B C} \Rightarrow \hat{\mathcal{Z}}=(\mathcal{A} \otimes \mathcal{I})\left(\mathcal{I} \otimes \mathcal{C}^{T}\right) \hat{\mathcal{B}}$,

where $\mathcal{I}$ is the identity matrix. We can rewrite Eq.(23) as follows

$0=\mathcal{S} \Lambda+\Lambda \mathcal{S}^{T}+2 \sum_{\xi} \mathcal{B}_{\xi} \Lambda \mathcal{B}_{\xi}^{T}+\mathcal{Q}$,

where

$\mathcal{Q}=2 \mathcal{C C}^{T}-\mathcal{V} \mathcal{V}^{T} \mathcal{S}^{-T}-\mathcal{S}^{-1} \mathcal{V} \mathcal{V}^{T}$

Previous properties allows for converting Eq.(30) into the following vectorized form

$0=\left[\mathcal{S} \otimes \mathcal{I}+\mathcal{I} \otimes \mathcal{S}+2 \sum_{\xi}\left(\mathcal{B}_{\xi} \otimes \mathcal{I}\right)\left(\mathcal{I} \otimes \mathcal{B}_{\xi}\right)\right] \hat{\Lambda}+\hat{\mathcal{Q}}$

and to get its solution as

$\hat{\Lambda}=-\left[\mathcal{S} \otimes \mathcal{I}+\mathcal{I} \otimes \mathcal{S}+2 \sum_{\xi}\left(\mathcal{B}_{\xi} \otimes \mathcal{I}\right)\left(\mathcal{I} \otimes \mathcal{B}_{\xi}\right)\right]^{-1} \hat{\mathcal{Q}}$

which can be calculated with standard numerical procedure (Gaussian elimination, factorizations and so on).

\section{References}

[1] Pop E 2010 Nano Res. 3147

[2] Hochbaum A I, Chen R, Delgado R D, Liang W, Garnett E C, Najarian M, Majumdar A and Yang P 2008 Nature 451 163-167

[3] Tavakoli A, Lulla K, Crozes T, Mingo N, Collin E and Bourgeois O 2018 Nature Comm. 94287

[4] Maldovan M 2012 Appl. Phys. Lett. 101113110

[5] Huang B W, Hsiao T K, Lin K H, Chiou D W and Chang C W 2015 AIP Advances 5053202

[6] Xu Z 2016 Theor. Appl. Mech. Lett. 6 113-121

[7] Li D, Wu Y, Kim P, Shi L, Yang P and Majumdar A 2003 Appl. Phys. Lett. 832934

[8] Maire J, Anufriev R and Nomura M 2017 Scientific Reports 741794

[9] Maire J, Anufriev R, Hori T, Shiomi J, Volz S and Nomura M 2018 Scientific Reports 84452

[10] Anufriev R, Gluchko S, Volz S and Nomura M 2019 Nanoscale 1113407 
[11] Zhuge F, Takahashi T, Kanai M, Nagashima K, Fukata N, Uchida K and Yanagida T 2018 J. Appl. Phys. 124065105

[12] Balandin A A 2011 Nature Mat. 10 569-581

[13] Wingert M C, Kwon S, Hu M, Poulikakos D, Xiang J and Chen R 2015 Nano Lett. 15 2605-2611

[14] Liu W and Asheghi M 2004 Appl. Phys. Lett. 843819

[15] Johnson J A, Maznev A A, Cuffe J, Eliason J K, Minnich A J, Kehoe T, Torres C M S, Chen G and Nelson K A 2013 Phys. Rev. Lett. 110025901

[16] Neogi S, Reparaz J S, Pereira L F C, Graczykowski B, Wagner M R, Sledzinska M, Shchepetov A, Prunnila M, Ahopelto J, Torres C M S and Donadio D 2015 ACS Nano 9 3820-3828

[17] Zaoui H, Palla P L, Giordano S, Cleri F, Verdier M, Lacroix D, Robillard J F, Termentzidis K and Martin E 2018 Int. J. Heat Mass Trans. 126 830-835

[18] Gomez-Heredia C L, Macias J, Ordonez-Miranda J, Ares O and Alvarado-Gil J J 2017 AIP Advances 7015032

[19] Huang R, Chavez I, Taute K M, Lukić B, Jeney S, Raizen M G and Florin E L 2011 Nature Physics 7 576-580

[20] Bae M H, Li Z, Aksamija Z, Martin P N, Xiong F, Ong Z Y, Knezevic I and Pop E 2013 Nature Comm. 41734

[21] Muñoz E, Lu J and Yakobson B I 2010 Nano Lett. 10 16521656

[22] Fourier J 1822 Théorie Analytique de la Chaleur (Paris: Didot)

[23] Brown R 1828 Philos. Mag. (New Series) 4 161-173

[24] Brown R 1829 Philos. Mag. (New Series) 6 161-166

[25] Einstein A 1905 Ann. Phys. (Leipzig) 17 549-560

[26] Einstein A 1906 Ann. Phys. (Leipzig) 19 371-381

[27] von Smoluchowski M 1906 Ann. Phys. (Leipzig) 21 756-780

[28] Perrin J B 1914 C. R. Acad. Sci. (France) 158 1168-1171

[29] Langevin P 1908 C. R. Acad. Sci. (France) 146 530-533

[30] Fokker A D 1914 Ann. Phys. 348 810-820

[31] Planck M 1917 Sitzber. Preuss. Akad. Wiss. 1917 324-341

[32] Chandrasekhar S 1943 Rev. Mod. Phys. 15 1-89

[33] Sekimoto K 1997 J. Phys. Soc. Jpn. 66 1234-1237

[34] Sekimoto K 2010 Stochastic Energetics (Berlin: Springer)

[35] Seifert U 2005 Phys. Rev. Lett. 95040602

[36] Seifert U 2012 Rep. Prog. Phys. 75126001

[37] Esposito M and Van den Broeck C 2010 Phys. Rev. E 82 011143

[38] Van den Broeck C and Esposito M 2010 Phys. Rev. E 82 011144

[39] Tomé T and de Oliveira M J 2010 Phys. Rev. E 82021120

[40] Tomé T and de Oliveira M J 2015 Phys. Rev. E 91042140

[41] Jarzynski C 1997 Phys. Rev. Lett. 782690

[42] Crooks G 1999 Phys. Rev. E 602721

[43] Collin D, Ritort F, Jarzynski C, Smith S B, Tinoco I and Bustamante C 2005 Nature 437 231-234

[44] EspositoM, Harbola U and Mukamel S 2009 Rev. Mod. Phys. 811665

[45] Rieder Z, Lebowitz J L and Lieb E 1967 J. Math. Phys. 8 1073

[46] Lepri S, Livi R and Politi A 2003 Physics Reports 377 1-80

[47] Gallavotti G 2008 The Fermi-Pasta-Ulam problem: a status report (Berlin Heidelberg: Springer)

[48] Fermi E, Pasta J and Ulam S 1965 Collected Papers (Chicago: University of Chicago Press)

[49] Benettin G and Ponno A 2011 J. Stat. Phys. 144 793-812

[50] Lepri S, Livi R and Politi A 1997 Phys. Rev. Lett. 781896

[51] Bonetto F, Lebowitz J L and Rey-Bellet L 2000 Mathematical Physics 2000 (London: Imperial College)

[52] Lepri S, Livi R and Politi A 2003 Phys. Rev. E 68067102

[53] Mai T, Dhar A and Narayan O 2007 Phys. Rev. Lett. 98 184301

[54] Dhar A 2008 Adv. Phys. 57457

[55] Casati G, Ford J, Vivaldi F and Visscher W M 1984 Phys. Rev. Lett. 521861

[56] Prosen T and Robnik M 1992 J. Phys. A 253449
[57] Sano M M and Kitahara K 2001 Phys. Rev. E 64056111

[58] Alonso D, Artuso R, Casati G and Guarneri I 1999 Phys. Rev. Lett. 821859

[59] Frenkel Y and Kontorova T 1938 Zh. Eksp. Teor. Fiz. 8 1340

[60] $\mathrm{Hu} \mathrm{B}$ and Yang L 2005 Chaos 15015119

[61] Pereira E and Falcao R 2006 Phys. Rev. Lett. 96100601

[62] Yang L 2002 Phys. Rev. Lett. 88094301

[63] Giardiná C, Livi R, Politi A and Vassalli M 2000 Phys. Rev. Lett. 842144

[64] Dhar A and Lebowitz J L 2008 Phys. Rev. Lett. 100134301

[65] Bolsterli M, Rich M and Visscher W M 1970 Phys. Rev. A 11086

[66] Rich M and Visscher W M 1975 Phys. Rev. B 112164

[67] Bonetto F, Lebowitz J L and Lukkarinen J 2004 J. Stat. Phys. 116783

[68] Reich K V 2013 Phys. Rev. E 87052109

[69] Gaspard P and Gilbert T 2008 New J. Phys. 10103004

[70] Bernardin C and Olla S 2005 J. Stat. Phys. 121271

[71] Basile G, Bernardin C and Olla S 2006 Phys. Rev. Lett. 96 204303

[72] Basile G and Olla S 2014 J. Stat. Phys. 1551126

[73] Basile G, Bernardin C, Jara M, Komorowski T and Olla S 2016 Thermal Transport in Low Dimensions 215-237 in Lecture Notes in Phys. 921 (Berlin: Springer)

[74] Giardinà C and Kurchan J 2005 J. Stat. Mech.: Theory Exp. 2005 P05009

[75] Dhar A, Venkateshan K and Lebowitz J L 2011 Phys. Rev. E 83021108

[76] Landi G T and de Oliveira M J 2013 Phys. Rev. E 87052126

[77] Landi G T and de Oliveira M J 2014 Phys. Rev. E 89022105

[78] Dhar A 2001 Phys. Rev. Lett. 865882

[79] Lemons D S 2002 An Introduction to Stochastic Processes in Physics (Baltimore: The Johns Hopkins University Press)

[80] Van Kampen N G 1981 Stochastic Processes in Physics and Chemistry (Amsterdam: Elsevier)

[81] Risken H 1989 The Fokker-Planck equation (Berlin: Springer Verlag)

[82] Coffey W T, Kalmykov Y P and Waldron J P 2004 The Langevin equation (Singapore: World Scientific)

[83] Fisk D L 1963 Quasi-martingales and stochastic integrals (Kent: Research monograph, Kent State University)

[84] Stratonovich R L 1966 SIAM J. Control 4362

[85] Itô K 1950 Nagoya Math. J. 135

[86] Hanggi P and Thomas H 1982 Phys. Rep. 88207

[87] Klimontovich Y L 1995 Statistical Theory of Open Systems (Dordrecht: Kluver Academic)

[88] Sokolov I M 2010 Chem. Phys. 375359

[89] Klein O 1921 Arkiv für Matematik, Astronomi och Fysik 161

[90] Kramers H A 1940 Physica 7284

[91] Manca F, Déjardin P M and Giordano S 2016 Annalen der Physik (Berlin) $\mathbf{5 2 8} 381$

[92] Bianco B, Moggia E, Giordano S, Rocchia W and Chiabrera A 2001 Il Nuovo Cimento 116B 155-167

[93] Giordano S 2019 Eur. Phys. J. B 92174

[94] Uhlenbeck G E and Ornstein L S 1930 Phys. Rev. 36823

[95] Wang M C and Uhlenbeck G E 1945 Rev. Mod. Phys. 17 323

[96] Lepri S 2016 Thermal Transport in Low Dimensions (Switzerland: Springer International Publishing) 